\title{
Investigation on Brushless DC Appropriateness to Direct-Drive Generator Wind Turbine
}

\author{
D. Vizireanu ${ }^{1}$, S. Brisset ${ }^{1}$, P. Brochet ${ }^{1}$, Y. Milet ${ }^{2}$, D. Laloy ${ }^{2}$ \\ ${ }^{1}$ L2EP - Ecole Centrale de Lille \\ Cité Scientifique, BP 48, 59651 Villeneuve d'Ascq Cedex, France \\ Phone/Fax number: +33(0) 3203354 54, e-mails: $\underline{\text { Darius.Vizireanu@ec-lille.fr, }}$ Stephane.Brisset@ec-lille.fr, $\underline{\text { Pascal.Brochet@ec-lille.fr }}$ \\ ${ }^{2}$ Framatome ANP \\ 27 Rue de l'Industrie, BP 189, 59573 Jeumont Cedex, France
}

\begin{abstract}
The paper studies the posibility to increase the power capability of direct-drive application. The system consists in a trapezoidal waveform PM brushless generator connected to the grid via a back-to-back concerter. Numerical model of the the system from the shaft of the generator until the DC bus circuit is setup. The studied parameters of the system are the electromagnetic torque ripple and the quality of the energy supplied to the grid. DC bus voltage adaption is proposed to avoid torque oscilltions and polyphased systems are proposed to reduce the low frequency harmonics of the power transferred to the grid.
\end{abstract}

Key words: direct-drive brushless dc generator, back-to-back converter, polyphased systems

\section{Introduction}

During last decades, an important development of permanent magnet machines has been observed, due to the improvement of the permanent magnet characteristics and the occurrence of new power electronic components. The magnets have allowed to eliminate the excitation and the slip rings, and to increase the specific power of the machines. The wind generators, the so-called direct-drive wind turbines, characterized by high torque and low speed parameters, represent one of the permanent magnet synchronous machines (PMSM) applications in the energy industry. For this type of application, an important quality criterion is the electromagnetic torque, and the interest is to minimize torque oscillations which cause lower mechanical stability, audible noise and accelerated ageing of the machine due to vibrations. The quality of energy supplied to the grid is the second quality criterion of the system.

The nowadays applications have limited power, in the domain of $1 \mathrm{MW}$ and efforts are concentrated to increase the power of PM direct-drive synchronous wind generators and its converter. For the PMSM, the idea of using trapezoidal waveforms is suggested by [1], where $30 \%$ increase volume power density is reported for a trapezoidal waveform machine comparing to a sinusoidal waveform machine. To increase the power capability of the converters, several solutions are suggested in the literature. Multi-level structures are used to increase the voltage capability. Parallel connection of converters is used to augment the current capability. This corresponds to an increase of the number of legs and consequently to polyphased machine. Polyphased structures offer the advantage of modularity [2], with immediate consequences for the fabrication process, assembly, transportation and maintenance.

The goal of this paper is to study the possibility to increase the power capability of a direct-drive wind generator using a trapezoidal waveform structure, presenting the assets and trying to propose solutions to overcome the drawbacks.

\section{System topology}

The architecture of the direct-drive PMSM wind generator consists of a discoid, one stator and two rotors, PMSM machine and a back-to-back converter (Fig.1).

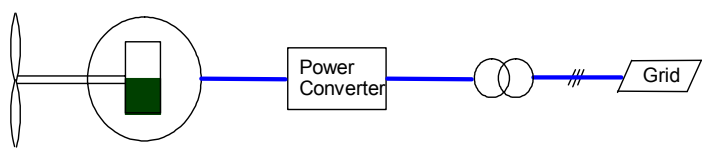

Figure 1. Direct-drive wind turbine system with PMSM

The back-to-back converter consists of a PWM rectifier, which controls the machine, and a PWM inverter for the grid connection. The intermediary circuit is represented by a DC bus capacitor that allows the decoupling of the two converters. The inverter controls also the DC bus voltage, which is imposed constant in order to protect the power transistors. The studied model is reduced: the invertertransformer-grid group is replaced by a DC voltage source connected in the DC bus circuit. The simplified model represents the considered system from the shaft of the generator until the DC bus, represented by a constant voltage source (Fig.2). For a constant DC voltage, the DC current gives an indication about the energy transferred via the DC bus, and consequently about the energy injected to the grid. The desired DC bus current waveform must have a small level of low frequency harmonics.

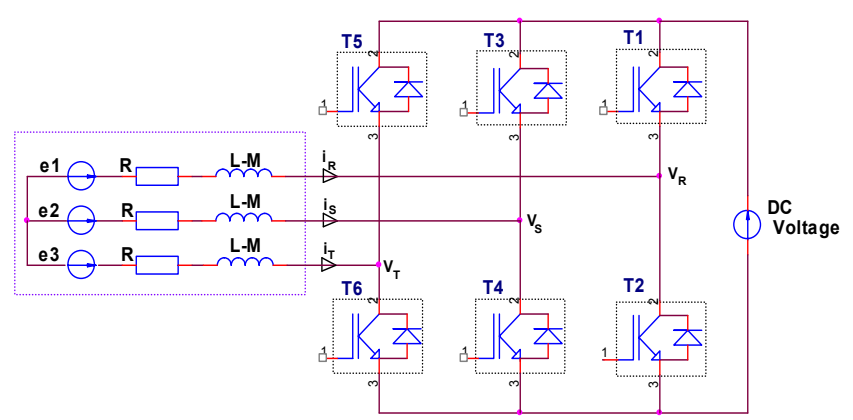

Figure 2. The simulation model for the machine and converter 


\section{Torque harmonics}

For a three-phase PMSM, without damping, the electromagnetic torque has the following expression:

$$
T_{\text {elmg }}=\frac{P_{\text {elmg }}}{\Omega}=\frac{1}{\Omega} \cdot \sum_{i=1}^{3} e_{i} \cdot i_{i}
$$

where $\Omega$ is the mechanical speed, $e_{i}$ is the e.m.f. corresponding to phase $i$, and $i_{i}$ is the phase current.

Using a Fourier series for the e.m.f. and imposing sinusoidal currents in phase with the e.m.f., the electromagnetic torque could be written as:

$$
T_{\text {elmg }}=\frac{3 E_{1} \cdot I}{2 \cdot \Omega}+\frac{3 \cdot I}{2 \cdot \Omega} \cdot \sum_{k=1}^{\infty}\left[\left(E_{6 k-1}+E_{6 k+1}\right) \cos (6 k \omega t)\right]
$$

It is easy to observe that the electromagnetic torque contains only $6^{\text {th }}$ or multiple by 6 harmonics. The torque harmonics are proportional with the current amplitude. Voltage harmonics $(6 k-1)$ and $(6 k+1)$ can be reduced using short-pitch winding techniques, but it is impossible to completely eliminate them. Controlling the machine's phase currents, torque ripple minimization can be realized by injecting current harmonics, but the current harmonics will be also found in the frequency spectrum of the DC bus current.

For the same volume of the machine, which allows same losses level, a higher electromagnetic torque is obtained by increasing the magnet width. The resulted e.m.f. will have the same peak value, but a higher value of the fundamental due to a higher $3^{\text {rd }}$ harmonic. However, using a wye connection, the $3^{\text {rd }}$ current harmonic will be eliminated and it won't influence the torque ripple.

When the currents contain odd harmonics, the electromagnetic torque becomes:

$$
\begin{aligned}
& T_{\text {elmg }}=\quad \frac{3}{2 \cdot \Omega}\left(E_{1} I_{1}+E_{3} I_{3}+E_{5} I_{5}+E_{7} I_{7}\right) \\
& +\frac{3}{2 \cdot \Omega}\left(E_{1} I_{5}+E_{1} I_{7}+E_{3} I_{3}+E_{5} I_{1}+E_{7} I_{1}\right) \cdot \cos (6 k \omega t) \\
& +\frac{3}{2 \cdot \Omega}\left(E_{5} I_{7}+E_{7} I_{5}\right) \cdot \cos (12 k \omega t)+\cdots
\end{aligned}
$$

The DC component of the torque can be increased using odd current harmonics in phase with the same order e.m.f. harmonics. Harmonics of the magnetic flux and current of the same order produce constant torque. Harmonics of the magnetic flux and current of different order produce pulsating torque. Equation (3) shows the interest of trapezoidal waveforms to increase the mean value of the torque.

The ideal e.m.f. waveform is a 120 electrical degrees trapezoidal form, while for the current the ideal shape is a 120 electrical degrees square wave. The electromagnetic torque, in this case, can be expressed as:

$$
T_{\text {elmg }}=\frac{2 \cdot E_{(t r)} \cdot I_{(t r)}}{\Omega}
$$

where $E_{(t r)}, I_{(t r)}$ are the peak values of the e.m.f., respectively the current.

However, in reality, the shape of the e.m.f. is not perfectly trapezoidal, and the current has not a perfect squared waveform. For the e.m.f., the width of the interval corresponding to the peak value depends on the width of the magnet. It could vary between $100^{\circ}$ and $150^{\circ}$. Deviation from the ideal forms of e.m.f. and current implies torque ripple.

\section{Numerical models}

First, a finite element model [3] is used to estimate the harmonic content of the e.m.f. If the shape of the magnet is defined, it is possible to use a finite element model to calculate the air-gap magnetic field. A magnet width of 0.9 reported to the pole pitch generates an e.m.f. as close as possible to the ideal one. An important flux leakage results due to the large magnet width. The finite element model allows the computation of the air-gap linkage inductance, the slots leakage inductance and the mutual inductances. The studied generator is an axial flux one, with one stator and two rotors. The symmetry and boundary conditions allow representing only one rotor and half of the stator (Fig. 3 top). The flux lines for no-load conditions are represented in the second picture of figure 3 .

A simulation model is used to study the behavior of the wind generator system, from the shaft of the generator until the DC bus. The model uses the e.m.f. waveforms and the machine parameters (resistances, inductances and rotor position) computed with the finite element model. Due to large air-gap and large slots, these inductances are practically linear. Rectangular currents are imposed in phase with the phase e.m.f. and the control is realized using hysteresis regulators. For the rectifier, the model allows also to introduce the parameters of each component (diode,
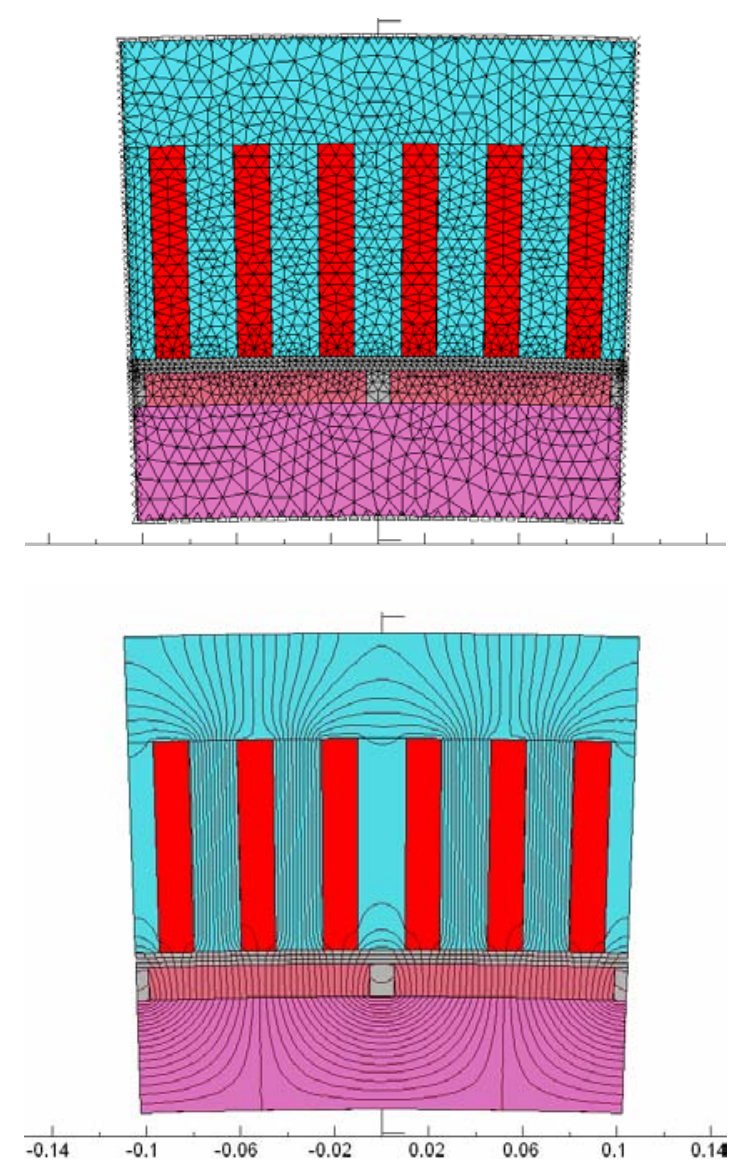

Figure 3. Finite elements model of the generator: the mesh (up) and the flux lines (bottom) 

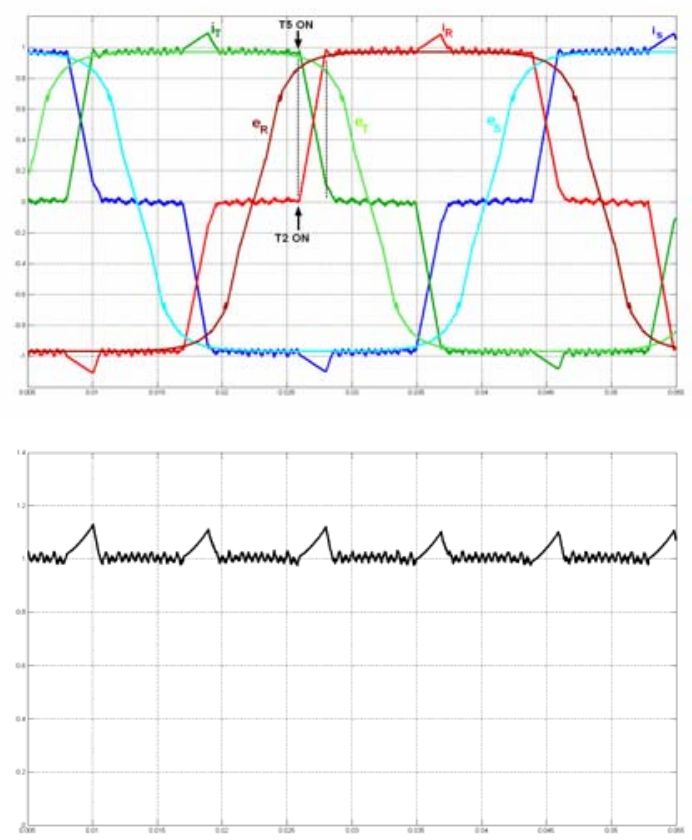

Figure 4. Phase currents (top) and electromagnetic torque (bottom) using un-adapted DC voltage

transistor, and snubber) which permits to estimate the converter losses. As mentioned by [4], [5] and [6], problems are encountered during the commutation process of the phase currents if the DC bus voltage has not the proper value, as illustrated in figure 4 , where the notations are according to figure 2. In this case the DC bus voltage was set to a value: $U_{D C}<4 \cdot E_{(t r)}$. The current $i_{R}$ reaches the peak value $\mathrm{I}_{(\mathrm{tr})}$ more rapidly than the current $i_{T}$ phase falls to zero, and consequently, the current $i_{S}$ is not constant during the commutation process. The amplitudes of peaks depend on the value of the DC bus voltage. The corresponding e.m.f is constant during the same period, and the electromagnetic torque will have an oscillation proportional to the current ripple. The analytical approach, described in the next section, explains this phenomenon.

\section{Analytical approach}

Consider the commutation interval where the current of the first phase increases up to the maximum value $I_{(t r)}\left(\mathrm{T}_{2}\right.$ $\mathrm{ON})$. Considering also that the current of the third phase is decaying to zero $\left(\mathrm{T}_{5} \mathrm{ON}\right)$ in the same period of time, the current of the second phase should remain approximately constant $i_{S}=-I_{(t r)}$ (D4 freewheeling diode) during the commutation. The equivalent circuit diagram is represented in figure 5. For the studied interval, all the three legs are conducting.

The electric potentials at the output of the generator are given by:

$$
\begin{aligned}
& v_{R}(t)=e_{R}-R \cdot i_{R}-L^{\prime} \cdot \frac{d i_{R}}{d t} \\
& v_{S}(t)=e_{S}-R \cdot i_{S}-L^{\prime} \cdot \frac{d i_{S}}{d t} \\
& v_{T}(t)=e_{T}-R \cdot i_{T}-L^{\prime} \cdot \frac{d i_{T}}{d t}
\end{aligned}
$$

where $L^{\prime}=L-M, e_{R}=e_{T}=-e_{S}=E_{(t r)}$.

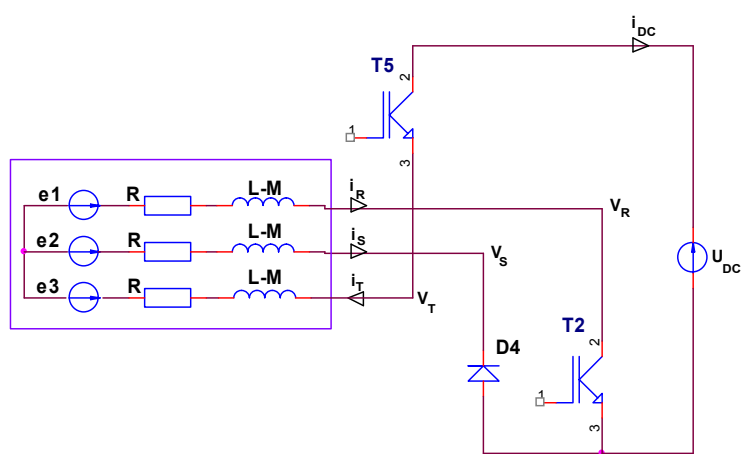

Figure 5. Equivalent circuit diagram for the studied commutation period

T2, T5 and D4 are conducting during the commutation period, and consequently, the voltages applied to the generator are:

$$
\begin{aligned}
& U_{R S}=v_{R}-v_{S}=e_{R}-e_{S}-R\left(i_{R}-i_{S}\right)-L^{\prime}\left(\frac{d i_{R}}{d t}-\frac{d i_{S}}{d t}\right)=0 \\
& U_{T S}=v_{T}-v_{S}=e_{T}-e_{S}-R\left(i_{T}-i_{S}\right)-L^{\prime}\left(\frac{d i_{T}}{d t}-\frac{d i_{S}}{d t}\right)=U_{D C}
\end{aligned}
$$

In the wye connection, $i_{R}+i_{S}+i_{T}=0$ and $\frac{d i_{R}}{d t}+\frac{d i_{S}}{d t}+\frac{d i_{T}}{d t}=0$. Neglecting the voltage drops across the power electronic devices, the phase currents equations become:

$$
\begin{aligned}
& R i_{R}+L^{\prime} \frac{d i_{R}}{d t}=\frac{1}{3}\left(U_{D C}+2 E_{(t r)}\right) \\
& R i_{T}+L^{\prime} \frac{d i_{T}}{d t}=-\frac{2}{3}\left(U_{D C}-E_{(t r)}\right)
\end{aligned}
$$

Using the notation $k_{U}=\frac{U_{D C}}{2 E_{(t r)}}$, which represents the ratio between the DC voltage and the peak line to line noload voltage, (7) becomes:

$$
\begin{aligned}
& R i_{R}+L^{\prime} \frac{d i_{R}}{d t}=\frac{U_{D C}}{3}\left(1+\frac{1}{k_{U}}\right) \\
& R i_{T}+L^{\prime} \frac{d i_{T}}{d t}=-\frac{2}{3} U_{D C}\left(1-\frac{1}{2 k_{U}}\right)
\end{aligned}
$$

The phase currents during commutation have the following expression:

$$
\begin{aligned}
& i_{R}(t)=\frac{U_{D C}}{3 R}\left(1+\frac{1}{k_{U}}\right)\left[1-\exp \left(-\frac{R}{L^{\prime}} t\right)\right] \\
& i_{T}(t)=I_{(t r)} \cdot \exp \left(-\frac{R}{L^{\prime}} t\right)-\frac{U_{D C}}{3 R} \cdot \frac{2 k_{U}-1}{k_{U}}\left[1-\exp \left(-\frac{R}{L^{\prime}} t\right)\right] \\
& i_{S}(t)=-I_{(t r)} \cdot \exp \left(-\frac{R}{L^{\prime}} t\right)+\frac{U_{D C}}{3 R}\left(1-\frac{2}{k_{U}}\right)\left[1-\exp \left(-\frac{R}{L^{\prime}} t\right)\right]
\end{aligned}
$$

Considering the time origin the beginning of the commutation, the current of the first phase has an initial value of $i_{R}(0)=0$ and a final value $i_{R}\left(t_{c}\right)=I_{(t r)}$, the 
commutation time can be calculated from (9):

$$
t_{c}=-\frac{L^{\prime}}{R} \ln \left(1-\frac{3 R I_{(t r)}}{U_{D C}} \cdot \frac{k_{U}}{k_{U}+1}\right)
$$

While $I_{(t r)}<<\frac{U_{D C}}{3 R}$, the current $i_{S}(t)$ is approximatively constant if $1-\frac{2}{k_{U}}=0$, i.e. $k_{U}=2$.

In this case, the peak-to-peak line electromotive force at nominal speed is equal to the half of the DC bus voltage:

$$
U_{D C}=4 \cdot E_{(t r)}
$$

\section{DC bus voltage adaptation}

The DC bus voltage is adapted in order to obtain a minimum torque ripple. Its value is imposed twice the peak value of the line-to-line no-load voltage.

Figure 6 presents the results obtained with the adapted value of the DC bus voltage. The current $i_{R}$ reaches the peak
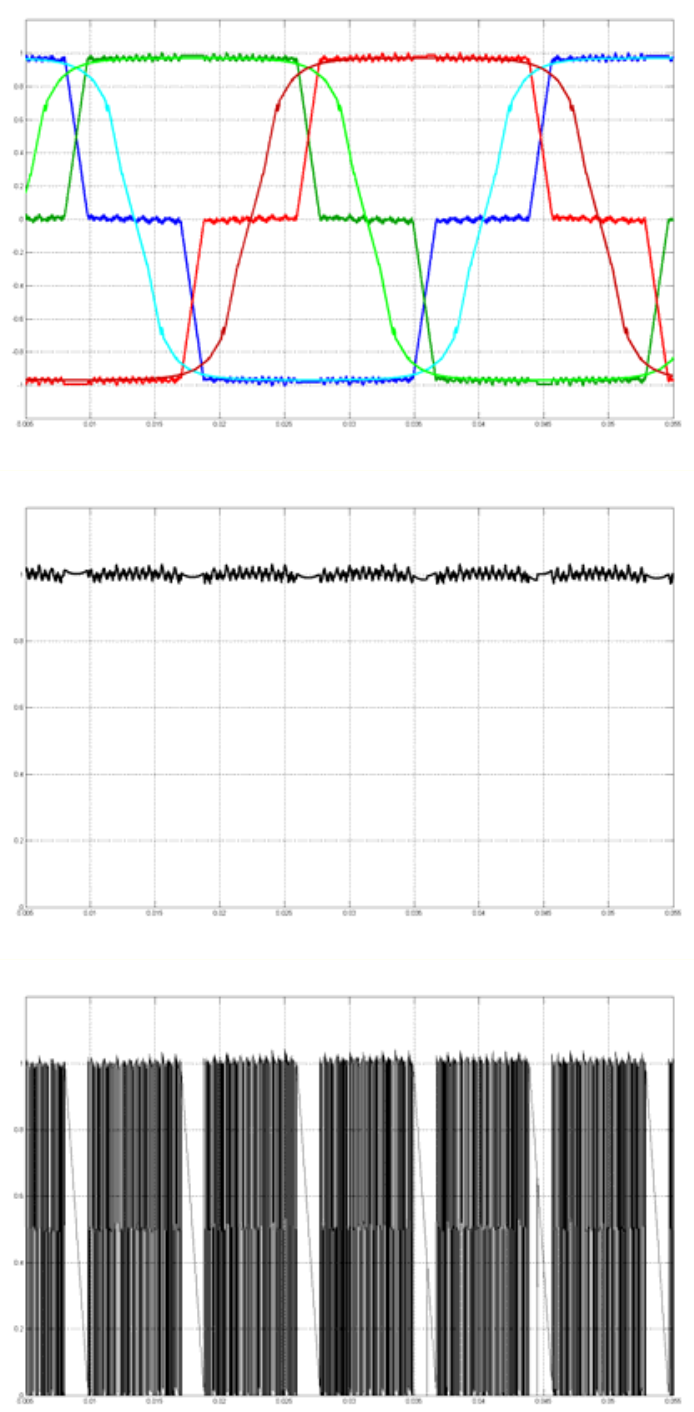

Figure 6. Numerical simulation using adapted DC bus voltage $\left(\mathrm{U}_{\mathrm{DC}}=4 \mathrm{E}_{(\mathrm{tr})}\right)$. Phase e.m.f. and currents (top), electromagnetic torque (middle) and DC bus current (bottom) value $\mathrm{I}_{(\mathrm{tr})}$ in the same time as the current $i_{T}$ phase falls to zero, and consequently, the current $i_{S}$ is approximatively constant during the commutation interval. Therefore, the torque is also constant during the phase commutation process.

For the studied commutation period, the DC bus current is $i_{D C}=i_{T}=-\left(i_{R}+i_{S}\right)$. The initial value is $I_{(t r)}$ and it decreases untill zero at the end of the interval. The DC current drops to zero six times per period (Fig. 6). If the DC bus voltage is considered constant during commutations, it means that energy transfer to the grid will have high oscillations. These oscillations have low frequencies ( $6 k$ order) and depend on the rotational speed (Fig. 7). Due to variable frequency operation, an additional filter is difficult to design.

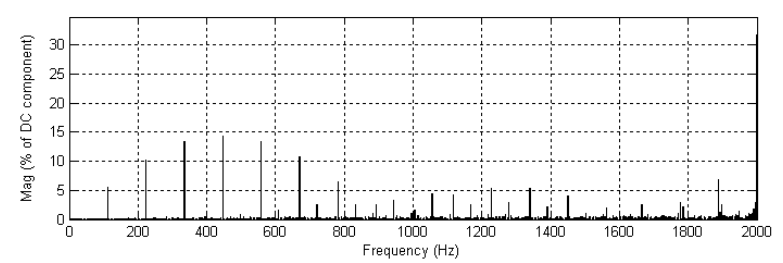

Figure 7. FFT analysis of the DC bus current for a 3-phase system

\section{Polyphased systems}

For the three phase system, the DC current presents high oscillations with a frequency six times the frequency of the phase currents. The envelope of the DC current waveform results from the positive part of each envelope of phase currents, excepting the commutation periods, when the DC current decrease from its maximum value $I_{(t r)}$ to zero. A solution to decrease the harmonics of the DC bus current is to increase the phase number. Generally, the interest is to use a number of phases multiple of three. The advantage consists in a possible modularity, which offers the advantage of easier assembly and transportation. The interface between the generator and the grid can be realized using classical 3-phase converters. At same rated power, a polyphased structure will have lower phase currents than a 3-phase one. In addition, 3phase stator cores can be used to replace their windings with six and nine-phase windings.

Theoretically, with an adapted phase shift, it is possible to reduce the oscillations amplitudes of the DC current, increasing in the same time their frequencies, which makes them easier to filter.

For a 6-phase system, it is not interesting to have a $0^{\circ}$ or $60^{\circ}$ phase shift between the stars because it generates synchronous oscillations for the DC currents of each rectifier. With a $30^{\circ}$ shift, the DC currents oscillations obtained will have a double frequency comparing to phase current, and the amplitudes of the oscillations are reduced, too. A theoretical graphical representation is done in figure 8 . The first and second graph represents the waveforms for the DC currents of the first and second rectifier. Assuming that the rectifiers are parallel connected through their DC bus; the third graph represents the total DC current. 


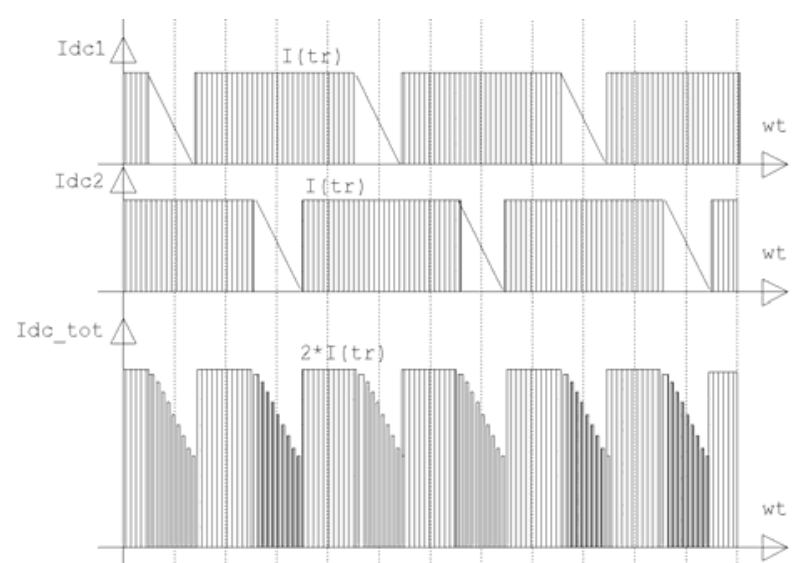

Figure $8.30^{\circ}$ shift 6-phase system - the DC bus currents for each rectifier and the total DC current

For a 9-phase system, a $20^{\circ}$ phase shift between the $\mathrm{Y}$ connections systems (Fig. 9) generates lowest DC total current oscillations.

Numerical simulations were used to validate this. The studied system consists on a 9-phase PM generator, with trapezoidal e.m.f. waveforms, connected to the grid via three back-to-back converters (Fig.10). The model is simplified: the group inverters-transformer-grid is replaced by a DC voltage source. The rectifiers are parallel connected via the DC bus. The armature currents are imposed in phase with the phase e.m.f. and the control is done using hysteresis regulators.

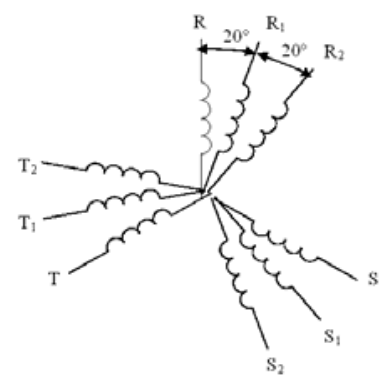

Figure 9 . Nine phase system with $20^{\circ}$ phase shift

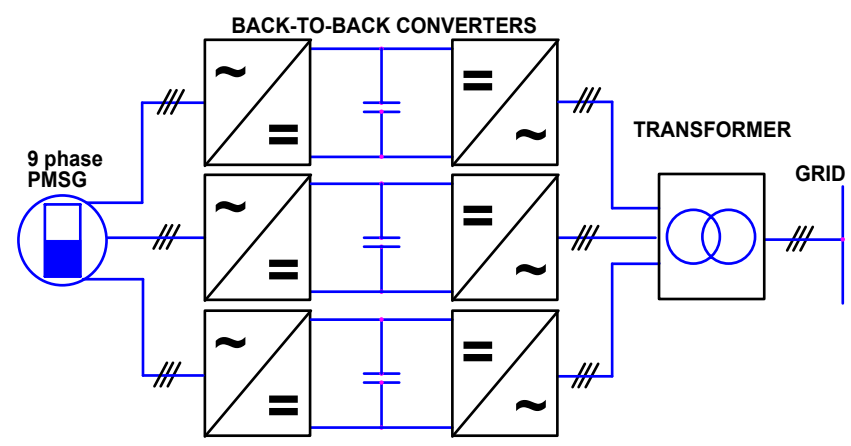

Figure 10. The structure of the 9-phase system

The operation of the generator is described by the following equation system:

$$
\left[v_{p h}\right]=\left[e_{p h}\right]-[R]\left[i_{p h}\right]-[L] \frac{d\left\lfloor i_{p h}\right\rfloor}{d t}
$$

where $\left[e_{p h}\right]=\frac{d}{d t}\left[\psi_{p h}\right]$ and $\left\lfloor\psi_{p h}\right\rfloor$ represents the matrix of the flux linkages generating the no-load e.m.f.s, $[L]$ is the inductances matrix which contains the leakage and mutual inductances.

$$
[L]=\left[\begin{array}{ccccccccc}
L & M_{1} & M_{1} & M_{2} & M_{3} & M_{4} & M_{3} & -M_{2} & -M_{4} \\
M_{1} & L & M_{1} & M_{4} & M_{2} & M_{3} & -M_{4} & M_{3} & -M_{2} \\
M_{1} & M_{1} & L & M_{3} & M_{4} & M_{2} & -M_{2} & -M_{4} & M_{3} \\
M_{2} & M_{4} & -M_{3} & L & M_{1} & M_{1} & M_{2} & M_{3} & M_{4} \\
-M_{3} & M_{2} & M_{4} & M_{1} & L & M_{1} & M_{4} & M_{2} & M_{3} \\
M_{4} & -M_{3} & M_{2} & M_{1} & M_{1} & L & M_{3} & M_{4} & M_{2} \\
M_{3} & -M_{4} & -M_{2} & M_{2} & M_{4} & -M_{3} & L & M_{1} & M_{1} \\
-M_{2} & M_{3} & -M_{4} & -M_{3} & M_{2} & M_{4} & M_{1} & L & M_{1} \\
-M_{4} & -M_{2} & M_{3} & M_{4} & -M_{3} & M_{2} & M_{1} & M_{1} & L
\end{array}\right]
$$

In the matrix of inductances, the components are: $L$ - the phase inductance;

$M_{I}$ - the mutual inductance between two phases of the same system;

$M_{2}$ - the mutual inductance between two phases of the different with a phase shift of $20^{\circ}$;

$M_{3}$ - the mutual inductance between two phases of the different with a phase shift of $140^{\circ}$;

$M_{4}$ - the mutual inductance between two phases of the different with a phase shift of $100^{\circ}$.

The results of the numerical simulation are presented in figures 11 and 12. Figure 11 presents the armature phase currents and the electromagnetic torque. The simulations were done with an adapted DC bus voltage. Therefore, the waveforms of the currents, respectively the torque waveform, contain only oscillations due to the hysteresis controllers.

The DC bus currents of each converter have the same waveform, but the oscillations are not synchronous (Fig. 12 $\mathrm{a}, \mathrm{b}$, and c). The total DC current (Fig. $12 \mathrm{~d}$ ) is the sum of the each DC bus. Fourier analysis of the total DC bus current reveals a lower spectrum of harmonics comparing to the three phase systems. In the case of 3-phase system, the DC
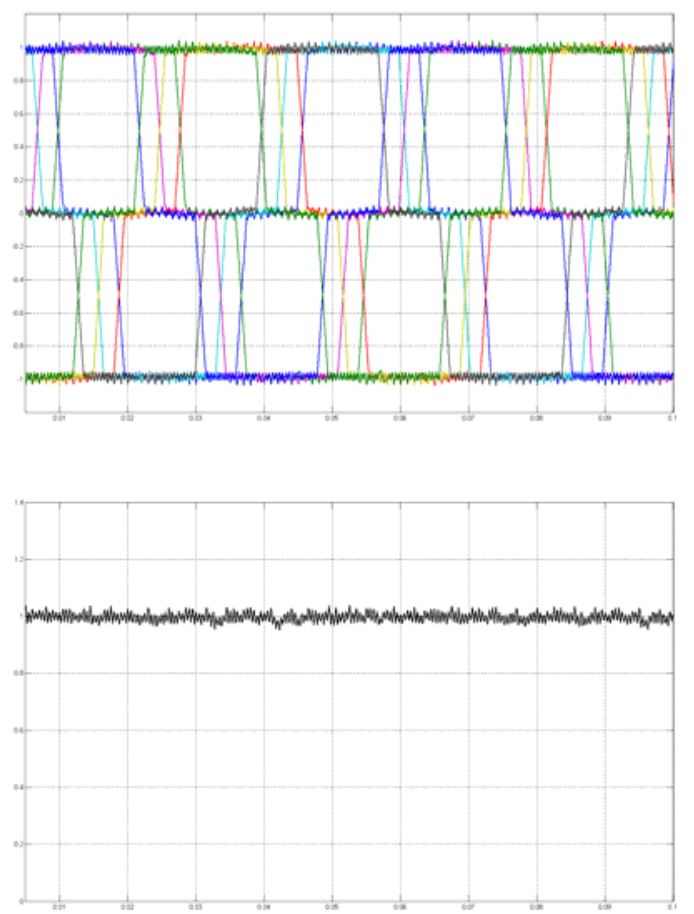

Figure 11. Numerical simulation for a $20^{\circ}$ phase shift 9-phase system - phase currents (top) and electromagnetic torque (bottom) 


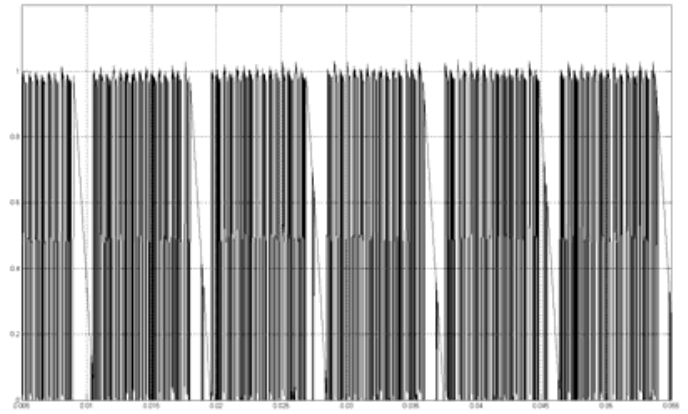

a) DC bus current for the $1^{\text {st }}$ rectifier

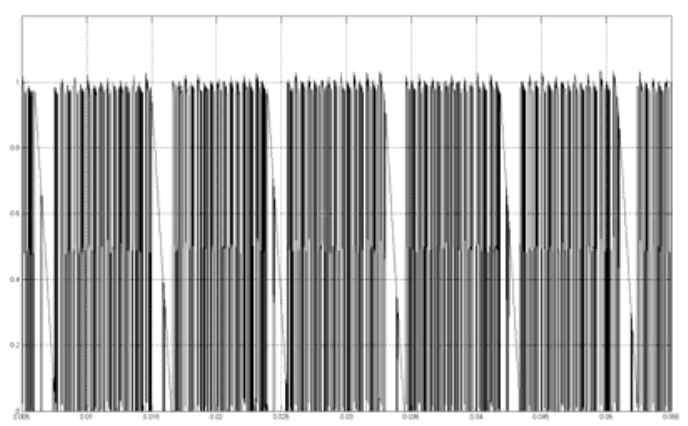

b) DC bus current for the $2^{\text {nd }}$ rectifier

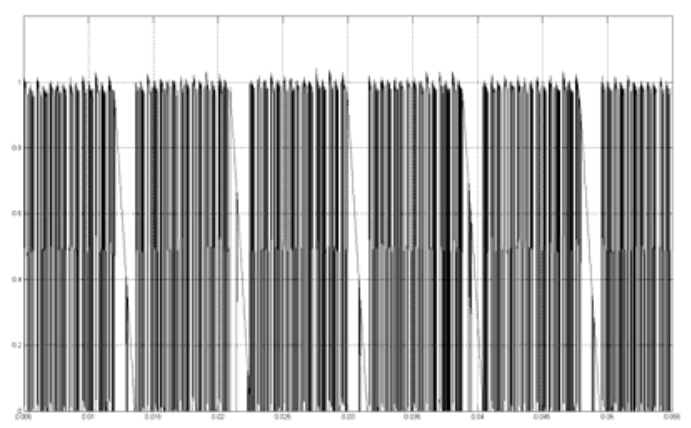

c) DC bus current for the $3^{\text {rd }}$ rectifier

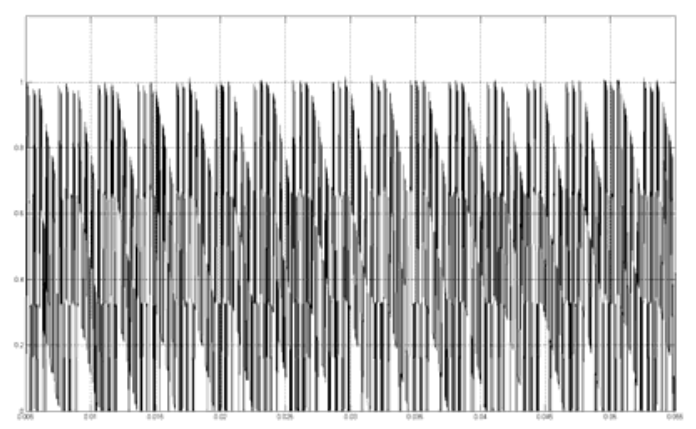

d) Total DC bus current

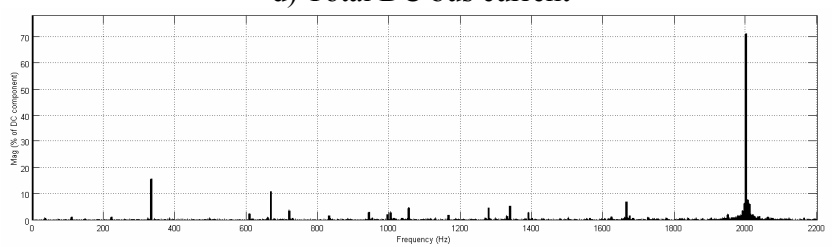

e)FFT analysis of the total DC bus current

Figure 12. Numerical simulation for a $20^{\circ}$ phase shift 9-phase system using adapted DC bus voltage $\left(\mathrm{U}_{\mathrm{DC}}=4 \mathrm{E}_{(\mathrm{tr})}\right)$ bus current contents a rich spectrum of $6 \mathrm{k}$ harmonics: $\mathrm{H} 6=$ $6 \%, \mathrm{H} 12=14 \%, \mathrm{H} 18=15 \%, \mathrm{H} 24=14 \%, \mathrm{H} 30=10 \%$. (Fig. 7 ). For the $20^{\circ}$ phase shift 9 -phase system, it easy to observe that the 6 and 12 order harmonics are vanished. The harmonics with the lowest frequencies found in the spectrum correspond to $\mathrm{H} 18=15 \%$ and $\mathrm{H} 36=10 \%$.

\section{Conclusions}

The paper studies the possibility of increasing the volume power density of PM generator designed for direct-drive wind turbine applications. The proposed solution is to use a trapezoidal waveform machine, which presents advantages, but also drawbacks comparing to the sinusoidal waveform machine. To overcome them, DC bus voltage adaptation is proposed to achieve minimum torque ripple, and polyphased structures are considered to reduce the low harmonics of the power to grid.

\section{References}

[1] Vizireanu D., Brisset S., Brochet P., Milet Y., Laloy D., " Study of Current and Electromotive Force Waveforms in Order to Improve the Performance of Large PM Synchronous Wind Generator", Proceedings of ICEM2004, Krakow, Poland;

[2] F. Caricchi, F. Crescimbini,, O. Honorati, "Modular, AxialFlux, Permanent-Magnet Motor for Ship Propulsion Drives", Electrical Machines and Drives Conference Record, 1997, IEEE International

[3] Vector Fields - Opera: http://www.vectorfields.com

[4] Jacek F. Gieras, Mitchell Wing, "Permanent Magnet Motor Technology", $2^{\text {nd }}$ Edition, Revised and Expanded

[5] Henderson J.R. Jr., Miller TJE, "Design of Brushless Permanent-Magnet Motors", Magna Physics Publishing et Calderon Press, Oxford 1994

[6] R. Carlson, M. Lajoie-Mazenc, J.C. dos S. Fagundes, "Analysis of Torque Ripple Due to Phase Commutation in Brushless dc Machines", IEEE Transactions on Industry Applications, Vol. 28, No. 3, May/June 1992

\section{Acknowledgement}

The work presented in this paper was done within FuturElec2 Archimed Project, supported by the CNRT in Electrical Engineering and Nord-Pas de Calais Region in France. 